\title{
Application of H-NMR metabolite fingerprinting and chemometrics for the authentication of Curcuma longa adulterated with Curcuma
}

\section{manga}

\author{
Anjar Windarsih ${ }^{1}$, Abdul Rohman $^{2 *}$, Respati Tri Swasono ${ }^{3}$ \\ ${ }^{1}$ Faculty of Pharmacy, Universitas Gadjah Mada, Yogyakarta. \\ ${ }^{2}$ Department of Pharmaceutical Chemistry, Faculty of Pharmacy, Universitas Gadjah Mada, Yogyakarta. \\ ${ }^{3}$ Department of Chemistry, Faculty of Mathematics and Natural Sciences, Universitas Gadjah Mada, Yogyakarta.
}

\begin{tabular}{l}
\hline ARTICLE INFO \\
\hline Article history: \\
Received on: 11/03/2018 \\
Accepted on: 27/04/2018 \\
Available online: 29/06/2018 \\
\hline Key words: \\
Chemometrics, Curcuma \\
longa, Curcumin, H-NMR, \\
TLC.
\end{tabular}

\begin{tabular}{l}
\hline ABSTRACT \\
Curcuma longa or turmeric is known to have many functions in the field of foodstuff, cosmetics, and traditional \\
medicines. The rhizome contains curcuminoid, especially curcumin which is believed to be the active compound \\
responsible for pharmacological activities. The authenticity of $C$. longa is very important to avoid adulteration practices. \\
The objective of this study was to develop thin layer chromatography (TLC) for curcumin analysis combined with \\
H-NMR metabolite fingerprinting and multivariate analysis for the authentication of $C$. longa powder. Determination \\
of curcumin in pure and adulterated powder of $C$. longa with Curcuma manga was performed using validated TLC \\
method with the composition of the mobile phase of toluene: glacial acetic acid ( $82: 18 \mathrm{v} / \mathrm{v}$ ) and silica gel $60 \mathrm{~F}_{254}$ as a \\
stationary phase with elution length $9 \mathrm{~cm}$. The contents of curcumin in pure powder from several regions were in the \\
range of $4.28-5.62 \%$, while those in adulterated were of $5.25-1.35 \%$. H-NMR metabolite fingerprinting combined with \\
chemometrics of principal component analysis (PCA) and orthogonal projections to latent structures-discriminant \\
analysis (OPLS-DA) using variables of the chemical shift was successfully used to classify the pure and adulterated \\
powder of C. longa.
\end{tabular}

\section{INTRODUCTION}

Curcuma longa L. or turmeric is one of the herbaceous plants which is widely cultivated in tropical regions especially in India and Southeast Asia. This rhizome has been known to have many functions in foodstuff, cosmetics, and traditional medicine. Numerous pharmacological activities of the rhizome have been reported such as antioxidant, anti-inflammatory, antibacterial, hepatoprotective, cardioprotective, and anticancer activity (Mohanty et al., 2004; Cousins et al., 2007). The rhizome has the rich contents in curcuminoid, especially curcumin which is believed to be the main active component. Curcumin content is very important because it was used as one of the parameters in quality control of $C$. longa (Cheng et al., 2010). High-quality

${ }^{*}$ Corresponding Author

Abdul Rohman, Department of Pharmaceutical Chemistry, Faculty of

Pharmacy, Universitas Gadjah Mada, Yogyakarta.

E-mail:abdul_kimfar@ugm.ac.id powder of C. longa will provide high-quality products. Because there are various products developed from turmeric and its demand is increasing, it is very important to ensure the authenticity of turmeric to avoid adulteration (Jurenka, 2009). Adulteration is a common practice in traditional medicine products. Adulteration poses a serious problem because it is related to the efficacy, safety, and quality of products. Turmeric is potential to be adulterated with other Curcuma species which are easy to obtain and have lower price such as Curcuma manga (Remya et al., 2004). Adulteration would affect curcumin content in the mixture because C. manga just has very low of curcumin. Therefore, it is very important to develop a fast and reliable method to detect the adulteration (Marikkar et al., 2001).

Several methods have been developed for authentication of turmeric powder such as thin layer chromatography (Pothitirat and Gritsanapan, 2005), high performance thin layer chromatography (Ashraf et al., 2012), high performance liquid chromatography (Jayaprakasha et al., 2002), and Fourier 
transform infrared spectroscopy (Rohman et al., 2015). Thin layer chromatography (TLC) has been developed for a long time for curcumin analysis in Curcuma species, especially C. longa powder (Paramasivam et al., 2009). However, the optimum TLC condition to obtain better resolution of curcumin separation and a good peak shape with minimum tailing still need to be explored. TLC offers many advantages for curcumin analysis, such as simple in preparation, use only small volume of organic solvent, can be used for qualitative and semiquantitative analysis, capable of analysis of several samples simultaneously, and required less time and cost (Phattanawasin et al., 2009). We developed a new method using mobile phase composition of toluene: glacial acetic acid $(82: 18 \mathrm{v} / \mathrm{v})$ to obtain better resolution and a good of peak shape. Therefore, we performed method validation to prove our proposed TLC method.

H-NMR spectroscopy appears as the sophisticated method in metabolite fingerprinting for plant natural products (van der Kooy et al., 2009). It offers some advantages over other analytical techniques because of its fast analysis, simple in sample preparation, high reproducibility, and it can simultaneously detect the diverse group of primary and secondary plant metabolites (Dai et al., 2010). Chemometrics of multivariate analysis has a great capability to analyze the huge data generated from H-NMR measurement (Kim et al., 2011). The application of H-NMR spectroscopy and multivariate analysis has been successfully applied to determine the species or origin of dandelion (Jung et al., 2011), Italian sweet cherries (Longobardi et al., 2013), and American Ilex (Kim et al., 2010). In addition, H-NMR spectroscopy and multivariate analysis have been successfully applied to differentiate between the pure and adulterated saffron (Petrakis et al., 2015). However, using literature searching, there is no metabolite fingerprinting study has been conducted to detect the adulteration of $\mathrm{C}$. longa powdered rhizome with $\mathrm{C}$. manga using H-NMR spectroscopy method.

Rafi et al. (2011) have differentiated C. longa, $C$. xanthorrhiza, and Zingiber cassumunar using TLC fingerprint analysis. However, they just performed qualitative TLC analysis by inspecting the resulted TLC spot of each species. In this study, we developed TLC as a new method for the authentication of C. longa by quantitatively determining curcumin content in both pure and adulterated $C$. longa with $C$. manga in various concentrations. Gad and Bouzabata (2017) have also developed H-NMR spectroscopy and chemometrics for quality control of turmeric. However, the model for the authentication of $C$. longa adulterated with $C$. manga has not been developed. In this study, the use of H-NMR spectroscopy-based metabolite fingerprinting combined with chemometrics of principal component analysis (PCA) and a more powerful technique, orthogonal projections to latent structures-discriminant analysis (OPLS-DA) were applied to differentiate between pure and adulterated powder of $C$. longa with C. manga.

\section{MATERIALS AND METHOD}

\section{Rhizome sample collection and preparation}

Rhizome samples of $C$. longa were collected from several regions in Yogyakarta and Central Java, while the rhizomes of $C$. manga were collected from Yogyakarta. Rhizomes were cleaned, chopped into small pieces, and air-dried. The dried rhizomes were ground into fine powder. The powder was used for analysis. The adulterated $C$. longa samples were prepared by adding $C$. manga in various proportions $(5,10,25,40,50$, and $75 \% \mathrm{wt} / \mathrm{wt})$.

\section{Standard and sample solution preparation}

Curcumin standard solution was prepared by dissolving $10 \mathrm{mg}$ of curcumin standard in $10 \mathrm{~mL}$ of methanol $(1 \mathrm{mg} / \mathrm{mL})$ as the stock solution.

For sample preparation, A-50 mg of pure and adulterated powder of $C$. longa was weighed accurately and put into $2 \mathrm{~mL}$ microtube. The powder was added with $1.5 \mathrm{~mL}$ methanol and then vortexed for 5 minutes. Subsequently, the samples were centrifuged for 5 minutes at $4000 \mathrm{rpm}$. The supernatant was used as the test solution.

\section{TLC instrumentations and conditions}

The standard and samples were spotted in the form of bands of width $5 \mathrm{~mm}$ with a Camag microliter syringe using a Camag Linomat V (CAMAG, Muttenz, Switzerland). The stationary phase used was precoated silica gel aluminum plate $60 \mathrm{~F}_{254}(20 \mathrm{~cm} \times 20 \mathrm{~cm}$ with $0.2 \mathrm{~mm}$ thickness; E.Merck, Darmstadt, Germany). The application rate was $150 \mathrm{~nL} / \mathrm{s}$ and the distance between two bands was $15 \mathrm{~mm}$. The mobile phase used was toluene: glacial acetic acid in a ratio $88: 18 \mathrm{v} / \mathrm{v}$. Plates were developed in ascending order in the chamber which was presaturated for 2 hours with the mobile phase. The elution length was $9 \mathrm{~cm}$ and the TLC was performed under temperature $25 \pm$ $2{ }^{\circ} \mathrm{C}$ and $\mathrm{RH} 60 \pm 5 \%$. After developing, the plate was dried and densitometric analysis was carried out at $427 \mathrm{~nm}$ using a Camag TLC scanner IV operated by WinCATS software.

\section{Method validation of thin layer chromatography}

Validation of TLC method was evaluated by assessing several performance characteristics namely specificity, linearity, accuracy, precision and sensitivity according to International Conference on Harmonization (ICH, 2005).

Specificity

The solutions of curcumin standard and sample were made and then spotted and eluated. The spectrum of standard and sample were observed. The spectrum and $\mathrm{Rf}$ (retardation factor) value of curcumin standard and curcumin in the sample must be identical.

\section{Linearity}

A $50 \mathrm{ppm}$ of curcumin standard solution was prepared from the stock solution. This solution was spotted in a different volume $(3,4,5,6$, and $7 \mu \mathrm{L})$ to obtain the concentrations of 150 , $200,250,300$, and $350 \mathrm{ng} / \mathrm{spot}$ of curcumin, respectively. The data of concentration versus peak area was observed. Linearity was evaluated by correlation coefficient $(\mathrm{R})$ value. Linearity with $\mathrm{R}$-value $\geq 0.997$ was acceptable for analysis.

\section{Accuracy}

Accuracy was performed using standard addition method. During accuracy analysis, $120 \mathrm{ng}, 150 \mathrm{ng}$, and $180 \mathrm{ng}$ of curcumin standard were added to the samples as the low, medium, and high-level concentration, respectively. The amounts of curcumin added were 
prepared from $60 \mathrm{ppm}, 75 \mathrm{ppm}$, and $90 \mathrm{ppm}$ of curcumin standard. The assay was performed in three replicates and recoveries value $(\%)$ of standard found in each concentration level were determined.

\section{Precision}

The intraday and interday precision were determined. Intraday precision was measured using samples in six replicates. The interday precision was performed by repeating the intraday assay in three different days. Precision was expressed as the percentage of coefficient variation $(\% \mathrm{CV})$.

\section{Sensitivity}

Sensitivity was evaluated by measuring limit of detection (LOD) and limit of quantification (LOQ). LOD and LOQ were calculated based on the following equations (LOD $=3.3 \sigma /$ slope of curcumin calibration curve; LOQ $=10 \sigma /$ slope of curcumin calibration curve). The LOD and LOQ found were performed using curcumin standard.

\section{Assay of curcumin content}

The sample test solutions were spotted on TLC plate, eluated, and detected. The percentage of curcumin content in each sample was determined by measuring the area under the curve (AUC) of each sample.

\section{Sample preparation for H-NMR measurement}

A-25 mg of pure and adulterated powder of C. longa was weighed and put into $2 \mathrm{~mL}$ centrifuge tube. The powder was added with $0.5 \mathrm{~mL}$ methanol D-4 (CD $\mathrm{OD})$ and $0.5 \mathrm{~mL} \mathrm{KH}_{2} \mathrm{PO}_{4}$ buffer $\mathrm{pH} 6.0$ in $\mathrm{D}_{2} \mathrm{O}$ contained TSP (trimethylsilyl propionic acid) $0.01 \%$. The $\mathrm{pH}$ was adjusted to 6.0 using NaOD $0.1 \mathrm{M}$. The mixture was vortexed for $1 \mathrm{~min}$, ultrasonicated for $20 \mathrm{~min}$, centrifuged at 13500 rpm for $10 \mathrm{~min}$, and then the supernatants $(800 \mu \mathrm{L})$ were transferred into NMR tubes.

\section{H-NMR measurement and multivariate analysis}

The H-NMR spectra were recorded on a $500 \mathrm{MHz}$ Jeol ECZ-R spectrometer. Each H-NMR spectrum acquired with the field strength of $11.74736 \mathrm{~T}(500 \mathrm{MHz})$, X_Offset $5.0 \mathrm{ppm}$, relaxation delay $5 \mathrm{~s}$ and 128 scans. The spectra were automatically and manually phase corrected. Baseline corrected was carried out using polynomial fit. The spectra were binned (bucketing) of equal width of $0.04 \mathrm{ppm}$ in the range of $0.00-10.00 \mathrm{ppm}$ excluding the regions of residual methanol (3.30-3.34 ppm) and water (4.71$5.10 \mathrm{ppm}$ ) using MestreNova 12.0.0.

\section{Data analysis}

Multivariate analysis was performed using PCA and OPLS-DA with Minitab 16 and SIMCA 14 software.

\section{RESULTS AND DISCUSSION}

\section{Method validation}

TLC used for quantitative analysis of curcumin was validated by determining specificity, linearity, accuracy, precision, and sensitivity. Specificity was measured by comparing the curcumin standard and samples for its Rf and UV spectra. A good resolved single spot of curcumin was observed at $\mathrm{Rf}$ value of $0.47 \pm 0.3$, either in standard or in evaluated samples (Figure 1). The densitogram of curcumin standard and curcumin in C. longa appeared at similar $\mathrm{Rf}$ value and a clear separation of curcumin from another component (demethoxycurcumin) in $C$. longa sample was obtained.

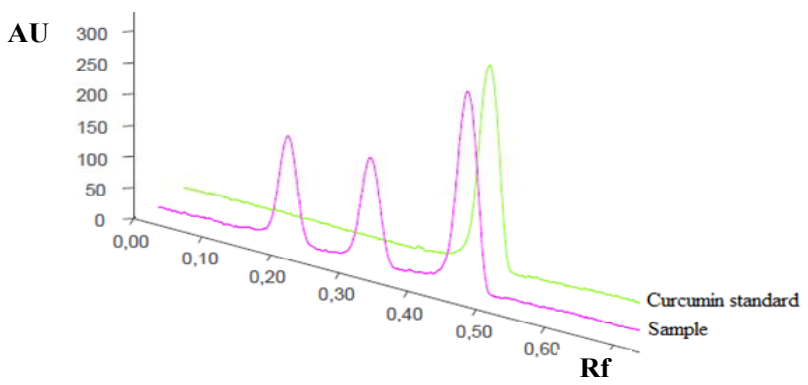

Fig. 1: The spectra of curcumin standard and curcumin in Curcuma longa sample.

Linearity was observed in the concentration range of 150-350 ng of curcumin standard. The calibration curve was linear with the regression equation of $y=26.815 x+1954.4$, and the determination and correlation coefficient were 0.097 and 0.9985, respectively (Figure 2). The developed method showed a linear calibration curve. According to Chan et al. (2004), the models with a determination coefficient $\left(\mathrm{R}^{2}\right) \geq 0.997$ have a good linearity. Accuracy is the closeness of agreement between an accepted reference value and the value found in the measurement (ICH, 2005). Accuracy is reported as percent recovery. The recoveries value were evaluated using standard addition method. Samples were added with curcumin standard in three different level, namely $120 \mathrm{ng}, 150 \mathrm{ng}$, and $180 \mathrm{ng}$, respectively. The recoveries of standard added were determined. The recoveries found were in the range of $99.06-101.10 \%$ (Table 1). The acceptance criteria for recoveries assay using concentration of analyte 60 ppm, 75 ppm, and 90 ppm was 90-107\% (Gonzalez and Herrador, 2007). Our results meet the requirement for recoveries acceptance criteria. Therefore, this method has a good accuracy.

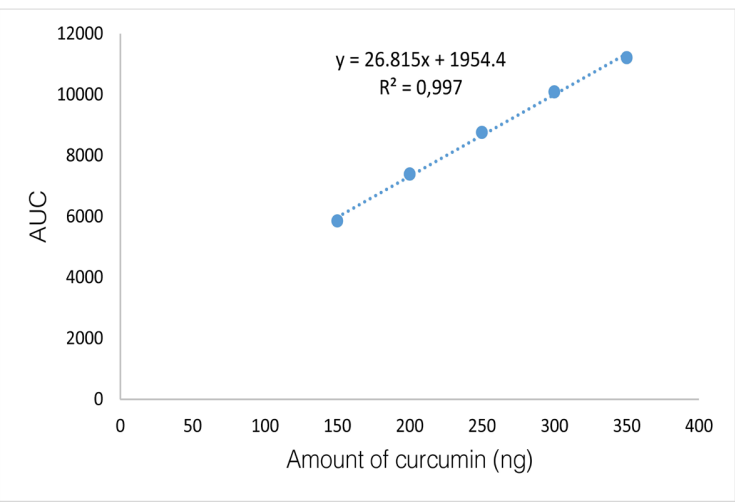

Fig. 2: Calibration curve of curcumin. 
Table 1: A recovery study of curcumin using standard addition method.

\begin{tabular}{ccccc}
\hline $\begin{array}{c}\text { Amount of } \\
\text { curcumin } \\
\text { added (ng) }\end{array}$ & $\begin{array}{c}\text { Amount of } \\
\text { curcumin } \\
\text { found (ng) }\end{array}$ & $\begin{array}{c}\text { Recovery } \\
(\%)\end{array}$ & $\begin{array}{c}\text { Mean } \\
\text { recovery (\%) }\end{array}$ & $\begin{array}{c}\text { RSD } \\
\text { (\%) }\end{array}$ \\
\hline 120 & 120.6116 & 100.51 & 100.01 & 0.88 \\
120 & 118.7917 & 98.99 & & \\
120 & 120.6340 & 100.53 & & \\
150 & 151.9411 & 101.29 & 101.10 & 0.27 \\
150 & 151.1803 & 101.79 & & \\
150 & 151.8068 & 101.20 & & \\
180 & 181.2605 & 100.70 & 99.06 & 1.43 \\
180 & 176.6735 & 98.15 & & \\
180 & 177.0166 & 98.34 & & \\
\hline
\end{tabular}

The intraday precision was measured using C. longa sample. The precision was expressed as \%RSD. The RSD values of intraday precision were $\leq 2 \%$ (Table 2 ). The interday precision was carried as in the intraday precision in three different days. The $\%$ RSD of interday precision was $\leq 2 \%$ (Table 2 ). For the precision assay, the acceptance criteria according to Horwitz for precision assay with analyte content $(\mathrm{x})$ of $1 \%<x \leq 10 \%$ was not more than $2.8 \%$ (Gonzalez and Herrador, 2007). Our results fulfilled the requirement for precision acceptance criteria. Therefore, this developed method has a good precision.

Sensitivity was observed by measuring limit of detection (LOD) and limit of quantification (LOQ) of the developed method. The LOD found was $16.46 \mathrm{ng}$ and the LOQ found was $49.89 \mathrm{ng}$. These results showed that this method has a good sensitivity. The low LOD and LOQ value showed the developed method has a good sensitivity. Curcumin contents were varied among several regions because metabolites formation was affected by their environmental conditions. The differences of the environmental condition among the regions may affect synthesis of metabolites including curcumin (Booker et al., 2014).

Table 2: Intraday and interday precision.

\begin{tabular}{|c|c|c|c|c|c|c|}
\hline \multirow{2}{*}{ Replication } & \multicolumn{2}{|c|}{ Day 1} & \multicolumn{2}{|c|}{ Day 2} & \multicolumn{2}{|c|}{ Day 3} \\
\hline & AUC & Amount (ng) & AUC & Amount (ng) & AUC & Amount (ng) \\
\hline 1 & 8133.2 & 230.4233 & 8024.4 & 226.3658 & 8045 & 227.1341 \\
\hline 3 & 7903.8 & 221.8684 & 7966.7 & 224.2141 & 8107 & 229.4462 \\
\hline 4 & 8057.7 & 227.6077 & 8107.2 & 229.4537 & 7971.7 & 224.4005 \\
\hline 6 & 8111.2 & 229.6028 & 8017.9 & 226.1234 & 8049.2 & 227.2907 \\
\hline Mean (ng) & & 226.2925 & & 228.9745 & & 229.2697 \\
\hline SD & & 3.54 & & 4.24 & & 3.90 \\
\hline RSD (\%) & & 1.57 & & 1.85 & & 1.70 \\
\hline
\end{tabular}

The TLC validated method was used to measure the curcumin contents of $C$. longa powdered rhizome from several regions and also the curcumin contents in the series of adulterated C. longa. The curcumin found from several regions of $C$. longa was around 4.28\%-5.62\% (Figure 3). C. longa from Gunung Kidul was chosen in making series of adulterated samples because it has the highest curcumin content. The curcumin found in the adulterated powder of C. longa with C. manga was around 5.25$1.35 \%$ (Figure 4). Curcumin content decreased as the adulterants concentration increased. Based on the previous report, the curcumin content in C. manga is very low, not more than $0.05 \%$ (Policegoudra et al., 2011), therefore, when they were mixed with pure $C$. longa powder, it will decrease the curcumin content in the mixtures. Therefore, the developed TLC method can be used for the quality control of $C$. longa to ensure its authenticity.

\section{H-NMR metabolite fingerprinting and chemometrics of multivariate analysis}

Metabolite fingerprinting can be understood as the chemical patterns resulted from analytical machine output which contains many information, for example, the H-NMR spectra resulted from the measurement. H-NMR metabolite fingerprinting provides about 100-1000 variables that will be very useful for further analysis (Verpoorte et al., 2007). Metabolite fingerprinting involved sorting dataset and then each sample was classified. Chemometrics of multivariate analysis is a statistical method that can manage the complex data generated from H-NMR spectra. It is very useful in metabolite fingerprinting analysis. The spectra of $C$. longa and $C$. manga showed a specific spectrum for each sample (Figure 5a and 5b). There are some differences in certain chemical shift regions. These differences were caused by their metabolite contents which were different for each Curcuma species. C. longa has more signals with higher intensities in the regions of 0.00-3.00 ppm, while C. manga just had a few signals with low intensities. In the regions of 5.50-10.00 ppm, C. longa also has more signals and higher intensities than C. manga, but there were no signals after the region of $8.00 \mathrm{ppm}$. C. manga still have signal in the regions of $8.50 \mathrm{ppm}$ and $9.50 \mathrm{ppm}$. Regions of 0.30-3.00 ppm, 3.00-6.00 ppm, and 6.00-10.00 ppm were associated with essential oils/ fatty acid, carbohydrates, and aromatic compounds, respectively (Booker et al., 2014). 


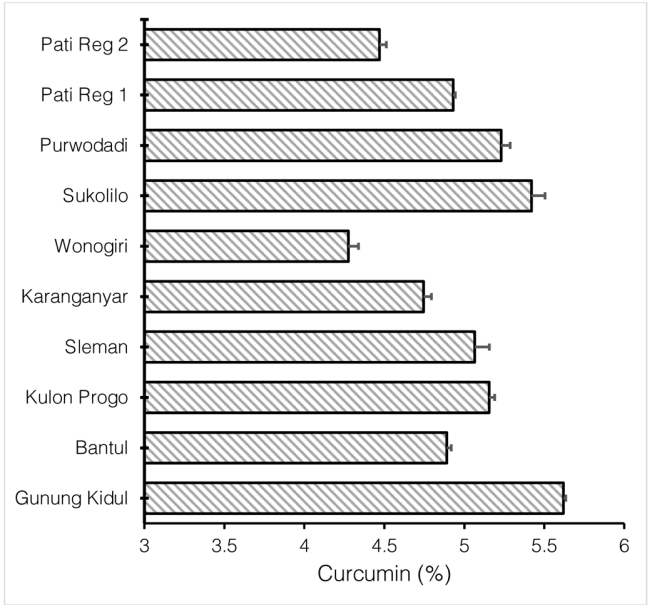

Fig. 3: Curcumin content in Curcuma longa from several regions.

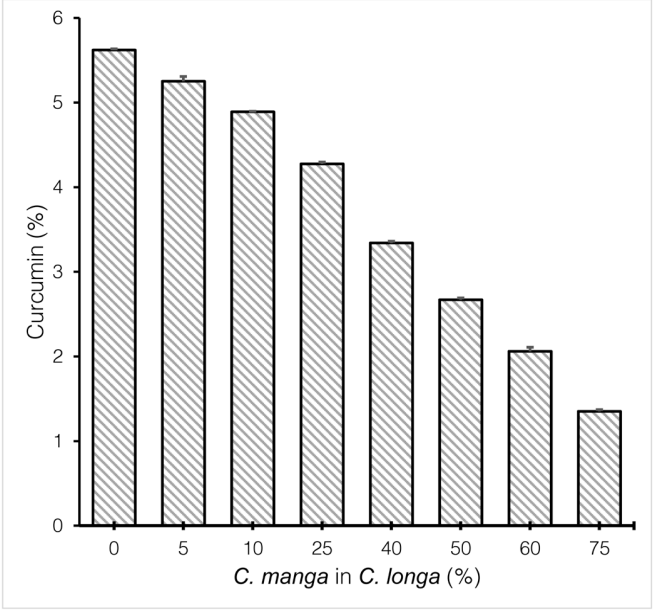

Fig. 4: Curcumin content in adulterated Curcuma longa with Curcuma manga.
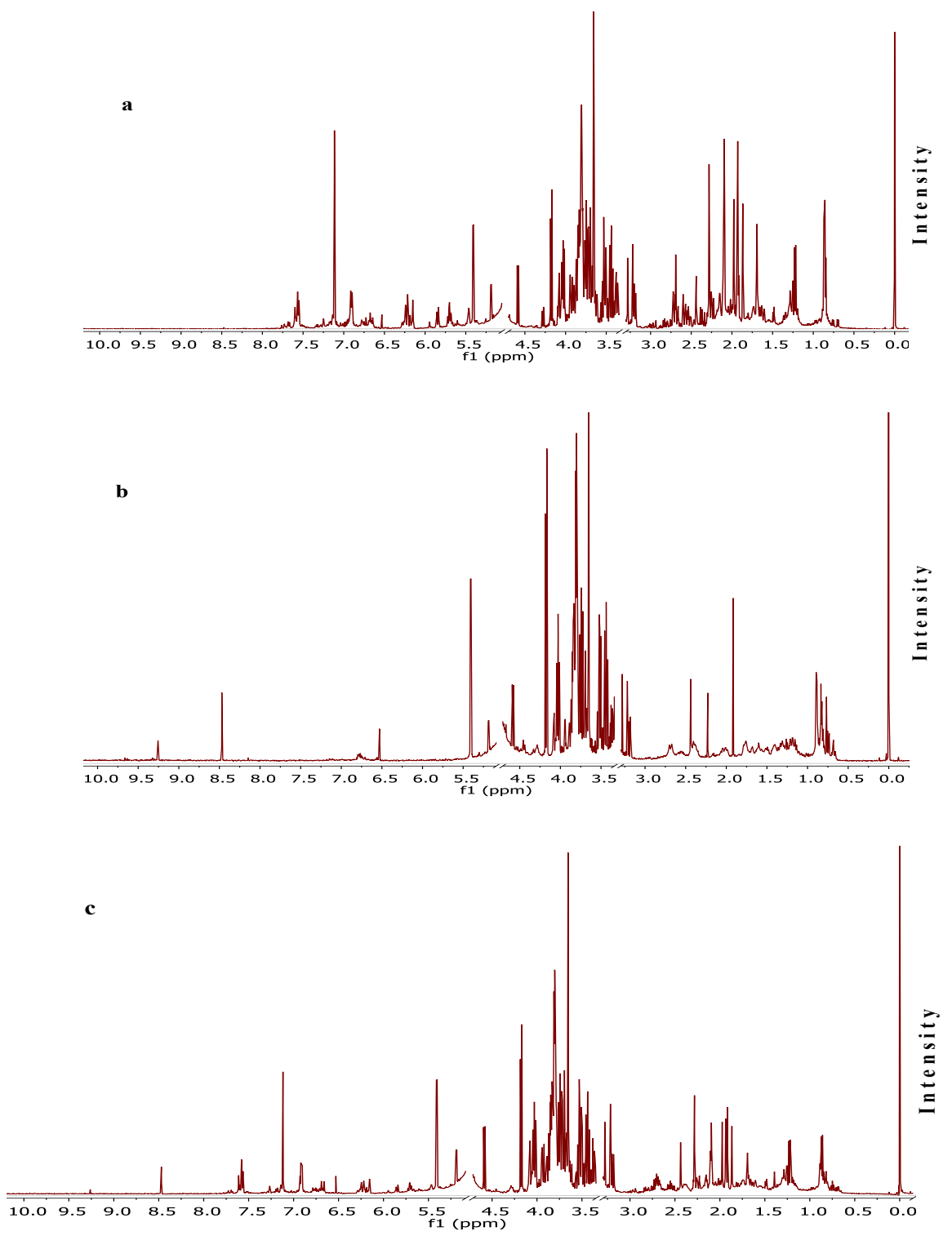

Fig. 5: H-NMR spectra of pure Curcuma longa (a), pure Curcuma manga (b), and adulterated Curcuma longa (50\%) with Curcuma manga (c). 
Curcuminoid is an example of metabolite contents in Curcuma species that will affect the profile of H-NMR spectra. Curcumin signals appeared in the regions of $7.28 \mathrm{ppm}$ (singlet), $3.90 \mathrm{ppm}$ (singlet), and $7.22 \mathrm{ppm}$ (doublet), while demethoxycurcumin signals appeared in the regions of 5.89 ppm (singlet), $3.94 \mathrm{ppm}$ (singlet), and $6.92 \mathrm{ppm}$ (doublet) (Awin et al., 2016). Because C. manga just has very low contents of curcuminoid, the signals of curcuminoid appeared at lower intensities compared to $C$. longa. Therefore, it makes the spectra of $C$. longa had more signals with higher intensities in the regions of 6.00-8.00 ppm than in C. manga. The spectra of adulterated $C$. longa with $50 \%$ of $C$. manga showed a similar pattern with the spectra of pure $C$. longa (Figure $5 \mathrm{c}$ ). It is difficult to distinguish by inspecting the spectra of pure and adulterated C. longa visually. Therefore, multivariate analysis is needed to resolve it.

PCA can be used to differentiate between pure $C$. longa and pure C. manga. The score plot of C. longa and C. manga appeared in the different area (Figure 6). Principal component analysis (PCA) is one of the multivariate analysis that can be used to classify and differentiate among a group of samples without any knowledge of their membership. Moreover, PCA also can be used to classify between pure and adulterated powder of C. longa. PCA was successfully distinguish between pure and adulterated powder of $C$. longa with $C$. manga in various proportions except in $5 \%$ of adulterant concentration (Figure 7 ). On the other hand, OPLS-DA is a supervised pattern recognition which allowed more powerful for classification compared to PCA. OPLS-DA allowed better separation of $C$. longa and adulterated $C$. longa with $C$. manga. All the series of adulterants were clearly separated from C. longa (Figure 8). OPLS-DA showed a good of fit $(\mathrm{R} 2 \mathrm{X}=0.912$, $\mathrm{R} 2 \mathrm{Y}=0.795)$ and good predictivity $(\mathrm{Q} 2=0.711)$. OPLS-DA was successfully distinguish between pure and adulterated $C$. longa with $C$. manga even in 5\% of adulterant concentration (Figure 8). Validation of OPLS-DA by permutation test showed that the OPLS-DA model is robust and credible. Therefore, the combination of H-NMR spectroscopy and multivariate analysis method become a powerful method for the authentication of $C$. longa.

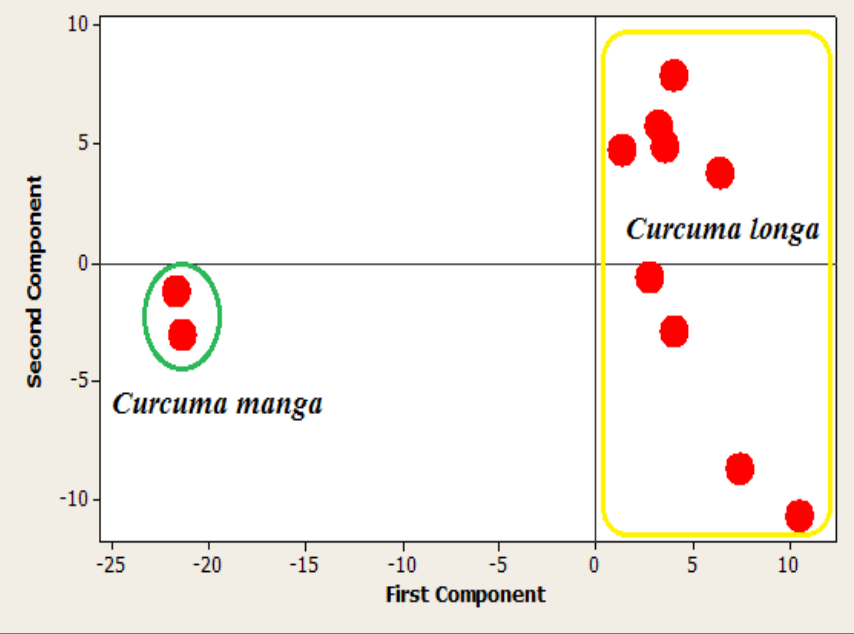

Fig. 6: PCA score plot of Curcuma longa and Curcuma manga.

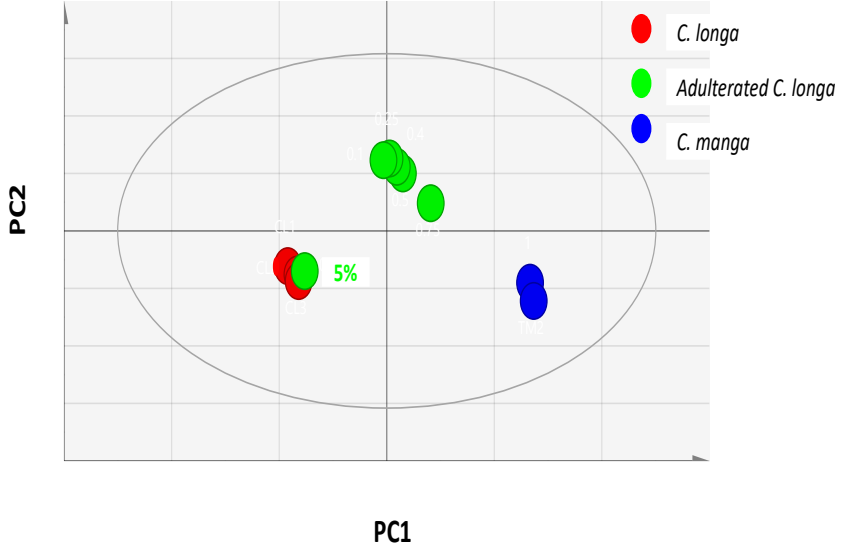

Fig. 7: PCA score plot of pure and adulterated Curcuma longa with Curcuma manga.

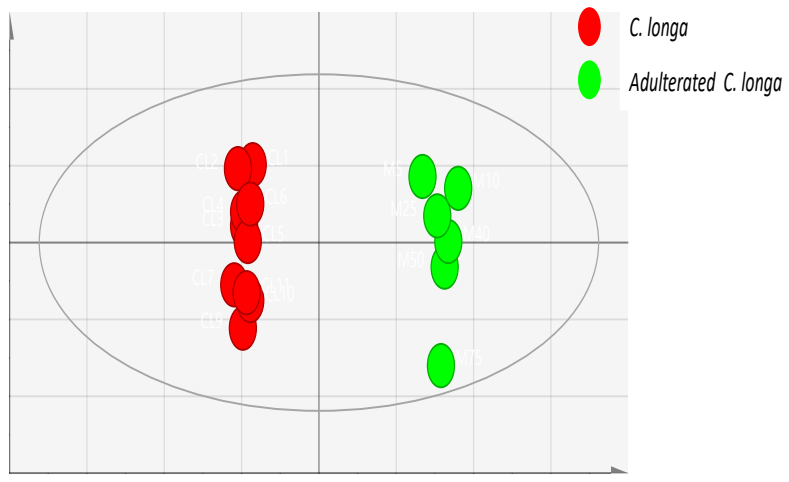

Fig. 8: OPLS-DA score plot of Curcuma longa from several regions and adulterated Curcuma longa with Curcuma manga.

\section{CONCLUSION}

In conclusion, the developed thin layer chromatography method for the estimation of curcumin content in pure and adulterated powder can be used for routine analysis of curcumin with good reproducibility. H-NMR spectroscopy method combined with chemometrics of PCA and OPLS-DA was a powerful method in metabolite fingerprinting and was confirmed successfully for classifying between pure and adulterated Curcuma longa powder with Curcuma manga.

\section{ACKNOWLEDGMENT}

The authors thankful to Faculty of Pharmacy Universitas Gadjah Mada, Indonesian Endowment Fund for Education Scholarship (LPDP) and Integrated Research and Testing Laboratory Universitas Gadjah Mada.

\section{REFERENCES}

Ashraf K, Mujeeb M, Ahmad A, et al. Validated HPTLC analysis method for quantification of variability in content of curcumin in Curcuma longa L (turmeric) collected from different geographical region of India. Asian Pac J Trop Biomed, 2012; 2:S584-S588.

Awin T, Mediani A, Maulidiani, et al. Phytochemical profiles and biological activities of Curcuma species subjected to different drying methods and solvent systems: NMR-based metabolomics approach. Ind Crops Prod, 2016; 94:342-352. doi: http://dx.doi.org/10.1016/j. indcrop.2016.08.020. 
Booker A, Frommenwiler D, Johnston D, et al. Chemical variability along the value chains of turmeric (Curcuma longa): a comparison of nuclear magnetic resonance spectroscopy and high performance thin layer chromatography. J Ethnopharmacol, 2014; 152:292-301. doi: http:// dx.doi.org/10.1016/j.jep.2013.12.042.

Chan CC, Lam H, Lee YC, Zhang XM. 2004. Analytical Method Validation and Instrument Performance Verification. Hoboken, New Jersey: Wiley Interscience, John Wiley and Sons, Inc.

Cheng J, Weijun K, Yun L, et al. Development and validation of UPLC method for quality control of Curcuma longa Linn.: Fast simultaneous quantitation of three curcuminoids. J Pharm Biomed Anal, 2010; 53:43-49. doi: http://dx.doi.org/10.1016/j.jpba.2010.03.021.

Cousins M, Adelberg J, Chen F, Rieck J. Antioxidant capacity of fresh and dried rhizomes from four clones of turmeric (Curcuma longa L.) grown in vitro. Ind Crops Prod, 2007; 25:129-135. doi: http://dx.doi. org/10.1016/j.indcrop.2006.08.004.

Dai H, Xiao C, Liu H, et al. Combined NMR and LC-DADMS analysis reveals comprehensive metabonomic variations for three phenotypic cultivars of Salvia Miltiorrhiza Bunge. J Proteome Res, 2010; 9:1565-1578. doi: http://dx.doi.org/10.1021/pr901045c.

Gad HA, Bouzabata A. Application of chemometrics in quality control of Turmeric (Curcuma longa) based on Ultra-violet, Fourier transform infra-red, and ${ }^{1} \mathrm{H}-\mathrm{NMR}$ spectroscopy. Food Chem, 2017; 237:857-864.

Gonzalez AG, Herrador MA. A practical guide to analytical method validation, including measurement uncertainty and accuracy profiles. Trends Anal Chem, 2007; 26:1-7.

ICH. 2005. Validation of analytical procedures: text and methodology Q2(R1). Int Conf Harmon Tech Requir Regist Pharm For Human Use.

Jayaprakasha GK, Jaganmohan RL, Sakariah KK. An improved HPLC method for the determination of curcumin, demethoxycurcumin and bisdemethoxycurcumin. J Agric Food Chem, 2002; 50:3668-3672.

Jung Y, Ahn YG, Kim HK, et al. Characterization of dandelion species using 1H-NMR- and GC-MS-based metabolite profiling. The Analyst, 2011; 136:4222-4231. doi: http://dx.doi.org/10.1039/c1an15403f.

Jurenka JS. Anti-inflammatory properties of curcumin, a major constituent of Curcuma longa: a review of preclinical and clinical research. Altern Med Rev J Clin Ther, 2009; 14:141-153.

Kim HK, Saifullah, Khan S, et al. Metabolic classification of South American Ilex species by NMR-based metabolomics. Phytochemistry, 2010; 71:773-784. doi: http://dx.doi.org/10.1016/j.phytochem.2010.02.001.

Kim H-S, Park SJ, Hyun S-H, et al. Biochemical monitoring of black raspberry (Rubus coreanus Miquel) fruits according to maturation stage by 1H NMR using multiple solvent systems. Food Res Int, 2011; 44:1977-1987. doi: http://dx.doi.org/10.1016/j.foodres.2011.01.023.

Longobardi F, VentrellaA, Bianco A, et al. Non-targeted 1H-NMR fingerprinting and multivariate statistical analyses for the characterisation of the geographical origin of Italian sweet cherries. Food Chem, 2013; 141:3028-3033. doi: http://dx.doi.org/10.1016/j.foodchem.2013.05.135.

Marikkar JMN, Lai OM, Ghazali HM, Man YBC. Detection of lard and randomized lard as adulterants in refined-bleached-deodorized palm oil by differential scanning calorimetry. J Am Oil Chem Soc, 2001; 78:1113-1119. doi: http://dx.doi.org/10.1007/s11746-001-0398-5.

Mohanty I, Singh Arya D, Dinda A, et al. Protective effects of Curcuma longa on ischemia-reperfusion induced myocardial injuries and their mechanisms. Life Sci, 2004; 75:1701-1711. doi: http://dx.doi. org/10.1016/j.lfs.2004.02.032.

Paramasivam M, Poi R, Banerjee H, Bandyopadhyay A. High-performance thin layer chromatographic method for quantitative determination of curcuminoids in Curcuma longa germplasm. Food Chem, 2009; 113:640-644. doi: http://dx.doi.org/10.1016/j.foodchem.2008.07.051.

Petrakis EA, Cagliani LR, Polissiou MG, Consonni R Evaluation of saffron (Crocus sativus $\mathrm{L}$.) adulteration with plant adulterants by (1)H NMR metabolite fingerprinting. Food Chem, 2015; 173:890-896. doi: http://dx.doi.org/10.1016/j.foodchem.2014.10.107.

Phattanawasin P, Sotanaphun U, Sriphong L. Validated TLCImage Analysis Method for Simultaneous Quantification of Curcuminoids in Curcuma longa. Chromatographia, 2009; 69:397-400. doi: http://dx.doi. org/10.1365/s10337-008-0893-y.

Policegoudra RS, Aradhya SM, Singh L. Mango ginger (Curcuma amada Roxb.) - a promising spice for phytochemicals and biological activities. J Biosci, 2011; 36:739-748.

Pothitirat W, Gritsanapan W. Quantitative analysis of curcumin, demethoxycurcumin and bisdemethoxycurcumin in the crude curcuminoid extract from Curcuma longa in Thailand by TLC-Densitometry. Mahidol Univ J Pharm Sci, 2005; 32:23-30.

Rafi M, Rohaeti E, Miftahudin A, Darusman LK. Differentiation of Curcuma longa, Curcuma xanthorrhiza and Zingiber cassumunar by thin layer chromatography fingerprint analysis. Indo J Chem, 2011; 11(1):71-74.

Remya R, Syamkumar S, Sasikumar B. Isolation and amplification of DNA from turmeric powder. Br Food J, 2004; 106:673678. doi: http://dx.doi.org/10.1108/00070700410558201.

Rohman A, Devi, Sudjadi, Nugroho A. Analysis of Curcumin in Curcuma longa and Curcuma xanthorriza Using FTIR Spectroscopy and Chemometrics. Res J Med Plant, 2015; 9:179-186. doi: http://dx.doi. org/10.3923/rjmp.2015.179.186.

van der Kooy F, Maltese F, Choi YH, et al. Quality control of herbal material and phytopharmaceuticals with MS and NMR based metabolic fingerprinting. Planta Med, 2009; 75:763-775. doi: http://dx.doi. org/10.1055/s-0029-1185450.

Verpoorte R, Choi YH, Kim HK. NMR-based metabolomics at work in phytochemistry. Phytochem Rev, 2007; 6:3-14. doi: http://dx.doi org/10.1007/s11101-006-9031-3.

How to cite this article:

Windarsih A, Rohman A, Swasono RT. Application of H-NMR metabolite fingerprinting and chemometrics for the authentication of Curcuma longa adulterated with Curcuma manga. J App Pharm Sci, 2018; 8(06): 075-081. 